\title{
Free radical activity in young adult cigarette smokers
}

\author{
M B Leonard, K Lawton, I D Watson, I MacFarlane
}

\begin{abstract}
Cigarette smoke is capable of generating free radicals. In this study markers of free radical activity (thiobarbituric acid reactivity, superoxide dismutase and glutathione peroxidase) were measured in young adult smoking and non-smoking volunteers. No significant differences were found in any of the free radical markers between the two groups. The number of leucocytes was elevated and a positive correlation was found between the number of leucocytes and thiobarbituric acid reactivity in the smokers. The results from this study suggest that any free radical activity generated from long term cigarette smoking in young adults is adequately scavenged.
\end{abstract}

( $(\mathcal{C}$ Clin Pathol 1995;48:385-387)

Keywords: Cigarette smoking, young adults, free radical markers.

Nitrogen dioxide, formed in cigarette smoke, can initiate polyunsaturated fatty acid autoxidation in membrane lipids with subsequent lipid peroxide formation. ${ }^{1}$ In addition to this effect, cigarette smoke may also be implicated in reduced activity of thiol dependent enzymes - for example, glutathione peroxidase ${ }^{1}$ and superoxide dismutase. ${ }^{2}$ Superoxide dismutase and glutathione peroxidase are components of the cellular defence system active against free radical attack.

Previous studies involving free radical activity measurements and smoking have usually involved older adults with established macrovascular disease and lung damage. ${ }^{2-5}$ None of these studies have objectively measured smoking load. The aims of the present study were to investigate the possible changes in free radical status induced by chronic cigarette smoking in a young healthy adult population. Urine cotinine, a metabolite of nicotine, was used to quantify the smoking load in these subjects.

\section{Methods}

Forty healthy adults (40 years old or less) with varied diets and no additional vitamin supplementation, medication, or excess alcohol consumption, participated in the study. There was no history of ischaemic heart disease, cerebro- or peripheral vascular disease in any of the subjects. The study was approved by the Ethics Committee of Aintree Hospitals.
Peripheral venous blood was collected into each of three tubes (Becton Dickinson vacutainer systems) containing EDTA (thiobarbituric acid reactivity, haemotological parameters), heparin (superoxide dismutase, glutathione peroxidase) and a serum separator gel (cholesterol, triglycerides, high density lipoprotein (HDL)). Blood samples were spun and processed immediately or stored at $-20^{\circ} \mathrm{C}$ until analysis. All blood samples were analysed within one week of storage. Random urine samples were collected without preservative for the estimation of creatinine and cotinine. Urine samples were stored at $-20^{\circ} \mathrm{C}$ until analysis. Cotinine results are expressed as $\mu \mathrm{g}$ cotinine/ mg creatinine.

\section{BIOCHEMICAL INVESTIGATIONS}

Cholesterol and triglycerides were measured by automated enzymatic methods on a Technicon RA-XT random access analyser using commercially available kits (Technicon Instruments, Basingstoke). HDL was estimated using a phosphotungstate precipitation method. Low density lipoprotein (LDL) was calculated by the Friedewald formula.

Cotinine was measured in the urine by radioimmunoassay using a double antibody technique (Diagnostic Products Corporation, Llanberis). Values are expressed as continine/ creatinine ratios $(\mu \mathrm{g} / \mathrm{mg})$. Urine creatinine was measured by the Jaffe reaction on a Technicon SMA.

Lipid peroxides were measured in plasma by high performance liquid chromatography. Quantification was achieved by comparing the product of the reaction between malondialdehyde (MDA) and thiobarbituric acid (TBA-RS) to a calibration curve of malondialdehyde prepared from 1,1,1-tetraethoxypropane. The results are expressed as $\mu \mathrm{mol} \mathrm{MDA} / \mathrm{l}(\mu \mathrm{mol} / \mathrm{l})$.

Superoxide dismutase was measured in erythrocytes using a commercially available kit (Randox Laboratories Ltd., Co. Antrim). This method uses xanthine and xanthine oxidase to generate superoxide radicals which react with a tetrazolium dye to form a red coloured compound. In the presence of superoxide dismutase this reaction is inhibited, therefore, the superoxide dismutase activity is measured by the extent of inhibition of the reaction.

Glutathione peroxidase was measured in erythrocytes using a commercially available kit (Randox Laboratories). The method follows the decrease in absorbance at $340 \mathrm{~nm}$ when 
Biochemical and haematological data for young adult smokers and non-smokers

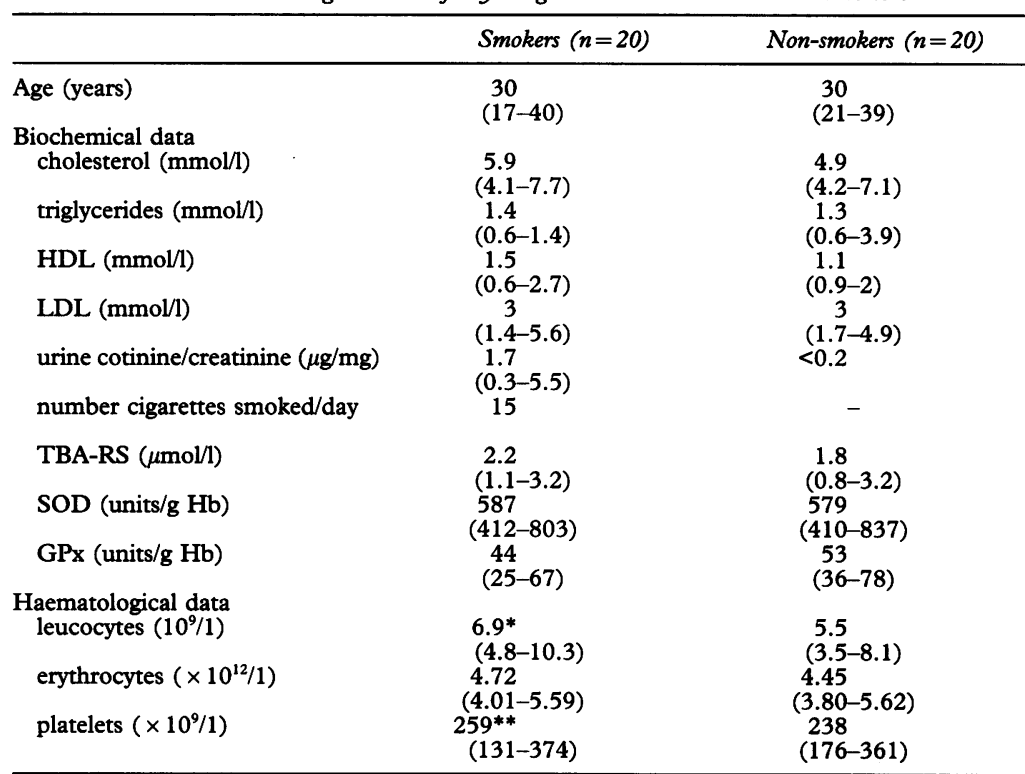

${ }^{*} \mathrm{p}=0.0070 v$ non-smokers; ${ }^{* *} \mathrm{p}=0.0890 v$ non smokers. TBA-RS $=$ thiobarbituric acid reactivity; $\mathrm{SOD}=$ superoxide dismuthase; $\mathrm{GPx}=$ glutathione peroxidase; $\mathrm{Hb}=$ haemoglobin.

oxidised glutathione is converted to glutathione via glutathione peroxidase.

Glutathione peroxidase and superoxide dismutase results are expressed as units enzyme activity/g haemoglobin.

\section{HAEMATOLOGY INVESTIGATIONS}

Leucocytes, erythrocytes and platelets were counted on a Sysmex K-1000 (Tao Medical Electronics Ltd., UK).

STATISTICAL ANALYSIS

All results are expressed as median and range. Data were analysed by the Mann-Whitney U test and Pearson correlation coefficients were used for correlation comparisons.

\section{Results}

Biochemical and haematological data are shown in the table.

\section{BIOCHEMICAL DATA}

Cotinine concentrations in non-smoking subjects were all lower than $0.2 \mu \mathrm{g} / \mathrm{mg}$ creatinine. No significant differences were found between cholesterol, triglycerides, HDL or LDL concentrations in the smokers and non-smokers.

No significant differences were found between thiobarbituric acid reactivity, superoxide dismutase, or glutathione peroxidase activities. No significant correlations were found between any of these free radical markers and the cotinine concentrations in the smokers.

\section{HAEMATOLOGICAL DATA}

The number of erythrocytes were similar in the smokers and non-smokers. However, the number of leucocytes was significantly increased $(p=0.0070)$ and the number of platelets marginally increased $(p=0.0890)$ in the smokers. A positive correlation was found between leucocytes and thiobarbituric acid reactivity in smokers $(r=0.575, p=0.0101)$.

\section{Discussion}

In the present study lipid peroxidation as measured by thiobarbituric acid reactivity was not elevated in smokers compared with nonsmokers. This is in agreement with Oen $e t a l,{ }^{3}$ who found no increases in lipid peroxide values due to smoking in populations older than 45 years. A study by Harats $e t a l^{4}$ on smokers aged less than 35 years indicated that smoking five to seven cigarettes during a 90 minute interval significantly increased lipid peroxidation. Protection against this increase was achieved by dietary supplementation of vitamin C or E for several weeks before their experiment. Non-smokers were not included in their study so a direct comparison between smoking and non-smoking lipid peroxidation values cannot be made.

The increased number of leucocytes and the correlation between leucocyte count and thiobarbituric acid reactivity in smokers from the present study may imply a correlation between altered oxidative metabolism and cigarette smoking. Nicotine, at concentrations found in the plasma of smokers, has been shown to enhance polymorphonuclear leucocyte influx into the lower airways of cigarette smokers. ${ }^{5}$ Stimulation of these leucocytes can result in increased production and release of superoxide free radicals with subsequent cellular and tissue damage. ${ }^{6}$ In addition, inactivation of lung antiprotease by free radical mediated oxidation of methionine groups has been proposed for the development of emphysema in smokers. ${ }^{7}$

Glutathione peroxidase and superoxide dismutase activities were unaffected by cigarette smoking in this study. Guemori et $a l^{2}$ also found no differences in erythrocyte superoxide dismutase activity in smokers, whilst Toth $e t a l^{8}$ found no differences in erythrocyte glutathione peroxidase activity in smokers although higher concentrations of glutathione and catalase were found.

The present study shows that any increase in oxidative stress that may be caused by chronic cigarette smoking in young adults clinically free of macrovascular disease and lung damage is insufficient to increase lipid peroxidation beyond that of non-smoking values. This may be because of increased activity of other antioxidant scavenging mechanisms not measured in this study or that the antioxidants measured have a large reserve and are not activated to maximum capacity at any one time.

In conclusion, it appears that any free radical intake acquired by long term cigarette smoking is adequately scavenged in young smokers.

1 Pryor WA, Tamura M, Church DF. ESR spin-trapping study of the radicals produced in Nox/Olefin reactions: A mechanism for the production of the apparently long-lived radcals in gas-phase cigarette smoke. $f$ Am Chem Soc 1984;

2 Guemori L, Artur Y, Herbeth B, Jeandel C, Cuny G, Siest G. Biological variability of superoxide dismutase, glutathione peroxidase, and catalase in blood. Clin Chem 1991;37: 1932-7.

3 Oen LH, Utomo H, Suyatna F, Hanafiah A, Asikin N. 
Plasma lipid peroxides in coronary heart disease. Int $\mathcal{f}$ Clin Pharmacol Ther Toxicol 1992;30:77-80.

4 Harats D, Ben-Naim M, Dabach Y, Hollander G, Havivi E, Stein $\mathrm{O}$, et al. Effect of Vitamin C and E supplementation on susceptibility of plasma lipoproteins to peroxidation induced by acute smoking. Atherosclerosis 1990;85:47-54.

5 Nowak D, Ruta U, Piascecka G. Nicotine increases human polymorphonuclear leukocytes chemotactic response-a possible additional mechanism of lung injury in cigarette smokers. Exp Pathol 1990;39:37-43.

6 Hoidal JR, Fox RB, LeMarbe PA, Perri R, Repine JE. Altered oxidative metabolic responses in vitro of alveolar macro- phages from asymptomatic cigarette smokers. Am Rev Respir Dis 1991;123:85-9.

7 Carp H, Miller F, Hoidal JR, Jaroff A. Potential mechanism of emphysema: alpha proteinase inhibitor recovered from lung of cigarette smokers contains oxidised methionine lungs of cigarette smokers contains oxidised methionine and has a decreased elastase inhibitory capacity. Proc Nat

8 Toth KM, Berger EM, Beehler CJ, Repine JE. Erythrocytes from cigarette smokers contain more glutathione and catalase and protect endothelial cells from hydrogen peroxide better than do erythrocytes from non-smokers. $A m$ Rev Respir Dis 1986;134:281-4.

\title{
Acquired protein $S$ deficiency in a patient with systemic lupus erythematosus causing central retinal vein thrombosis
}

\author{
H M Prince, P J Thurlow, R C Buchanan, K M A Ibrahim, P J Neeson
}

\section{Department of \\ Haematology, \\ Austin Hospital, \\ Heidelberg, \\ Victoria, Australia \\ H M Prince \\ P J Thurlow \\ P J Neeson}

Department of

Medicine

R C Buchanan

Department of

Haematology,

Repatriation General

Hospital,

Heidelberg, Victoria,

Australia

K M A Ibrahim

Correspondence to:

Dr H M Prince,

ABMT Program,

Toronto Hospital,

General Division,

200 Elizabeth Street,

mlw 2-036,

Toronto M5G 2C4,

Ontario, Canada.

Accepted for publication

25 August 1994

\begin{abstract}
A 16 year old girl with systemic lupus erythematosus (SLE) developed the rare complication of central retinal vein occlusion. Although classically a disease of older patients, it has been recognised in association with SLE but only in the presence of the lupus anticoagulant or antiphospholipid antibodies. The thrombosis occurred when free protein $S$ concentrations were transiently reduced and there was no family history or other known causes of reduced protein $S$ concentrations. No other prothrombotic risk factors were present.

(f Clin Pathol 1995;48:387-389)
\end{abstract}

Keywords: Protein S, systemic lupus erythematosus, central retinal vein thrombosis.

Central retinal vein occlusion (CRVO) is typically a disease of older patients, rarely occurring before the third decade $^{1}$ and is associated with either an underlying vascular disease or a procoagulant state. Hypertension, diabetes, vasculitides, and hyperlipidaemia are the most commonly associated vascular disorders while the recognised procoagulant states include thrombocytosis, polycythaemia, macroglobulinaemia, the oral contraceptive pill, or systemic lupus erythematosus (SLE). The latter, however, has only been associated with CRVO in the presence of the lupus anticoagulant (LAC) and/or antiphospholipid antibodies (APLA). ${ }^{2}$

LAC/APLA are associated with the increased thrombogenic potential of SLE but other factors have been implicated including abnormalities of the fibrinolytic system, factor VIII complex modifications, increased Von Wil- lebrand factor activity, acquired protein $\mathrm{C}$ deficiency, antithrombin III deficiency, and acquired prostoglandin $\mathrm{I}_{2}$ deficiency. More recently, the laboratory inter-relation between SLE, protein $S$ and $C 4 b$ binding protein (C4BP) has been recognised. ${ }^{3-6}$ However, this relation has not yet translated into a predictable increase in clinical thrombotic risk. Here, we present the case of a young patient with SLE, in whom LAC/APLA were absent but transiently low protein $S$ concentrations led to a predisposition to the development of a thrombosis of her central retinal vein.

\section{Case report}

A 16 year old, previously well, Malaysian girl presented five months earlier with immune thrombocytopenia and SLE. She was not taking any medication. The findings on examination were unremarkable with the exception of a malar rash and lower limb petechiae. A full blood count revealed a haemoglobin of $121 \mathrm{~g} / \mathrm{l}$, a total white cell count of $5 \cdot 8 \times 10^{9} / 1$ with a normal differential count and a platelet count of $5 \times 10^{9} / 1$. Her bone marrow was normocellular with adequate megakaryopoiesis. Normal investigation findings included Epstein-Barr virus and human immunodeficiency virus (HIV) serology, protein electrophoresis, and renal and liver function. Coagulation profile was normal including an activated partial thromboplastin time (APTT) of 36 seconds (normal 25-40). Antinuclear antibodies (ANA) were present in a speckled and nucleolar pattern with a titre of 1 in 10240 . Anti-Ro (SS-A) and anti-LA (SS-B) were demonstrated and antiDNA was $160 \mathrm{IU} / \mathrm{ml}$ (normal <4 IU/ml). Her thrombocytopenia responded to prednisolone $75 \mathrm{mg} /$ day, but she relapsed shortly afterwards 